\title{
Direct Regulation of TLR5 Expression by Caveolin-1
}

\author{
Jae Sung Lim Lim, $^{1,40}$, Kim Cuc Thi Nguyen ${ }^{1,2,10}$, Jung Min Han ${ }^{5,6,6}$, Ik-Soon Jang ${ }^{7}$, Claire Fabian ${ }^{8,9}$, \\ and Kyung $A$ Cho $0^{1,2,3,4, *}$
}

Toll-like receptor 5 (TLR5) is a specific receptor for microbial flagellin and is one of the most well-known receptors in the TLR family. We reported previously that TLR5 signaling is well maintained during aging and that caveolin-1 may be involved in TLR5 signaling in aged macrophages through direct interactions. Therefore, it is important to clarify whether caveolin-1/TLR5 interactions affect TLR5 expression during aging. To assess the effect of caveolin-1 on TLR5, we analyzed TLR5 expression in senescent fibroblasts and aged tissues expressing high levels of caveolin-1. As expected, TLR5 mRNA and protein expression was well maintained in senescent fibroblasts and aged tissues, whereas TLR4 MRNA and protein were diminished in those cells and tissues. To determine the mechanism of caveolin-1-dependent TLR5 expression, we examined TLR5 expression in caveolin-1 deficient mice. Interestingly, TLR5 mRNA and protein levels were decreased dramatically in tissues from caveolin-1 knockout mice. Moreover, overexpressed caveolin-1 in vitro enhanced TLR5 mRNA through the MAPK pathway and prolonged TLR5 protein half-life through direct interaction. These results suggest that caveolin-1 may play a crucial role in maintaining of TLR5 by regulating transcription systems and increasing protein half-life.

\section{INTRODUCTION}

Aging is an important risk factor that impairs tissue and cellular functions as well as promotes aging-related degenerative dis-

\footnotetext{
${ }^{1}$ Department of Biochemistry, ${ }^{2}$ Center for Creative Biomedical Scientists, ${ }^{3}$ Research Institute of Medical Sciences, Chonnam National University Medical School, Gwangju 501-746, Korea, ${ }^{4}$ Clinical Vaccine R\&D Center, Chonnam National University Hwasun Hospital, Hwasun 519-809, Korea, ${ }^{5}$ Department of Integrated OMICS for Biomedical Science, Yonsei University, Seoul 120-749, Korea, ${ }^{6}$ College of Pharmacy, Yonsei University, Incheon 406-840, Korea, ${ }^{7}$ Division of life Science, Korea Basic Science Institute, Daejeon 305-333, Korea, ${ }^{8}$ Department of Immunology, Fraunhofer Institute for Cell Therapy and Immunology (IZI), ${ }^{9}$ Translational Center for Regenerative Medicine (TRM), University of Leipzig, 04103 Leipzig, Germany, ${ }^{10}$ These authors contributed equally to the work.

*Correspondence: kacho@chonnam.ac.kr
}

Received 3 August, 2015; revised 24 September, 2015; accepted 25 September, 2015; published online 26 November, 2015

Keywords: aging, caveolin-1, senescence, toll-like receptor 5 eases (Campisi, 2013; Campisi et al., 2011). The aging process is complex with significant changes in many different molecules, and some of these molecules can be used as aging indicators (Menni et al., 2013). Several aging markers have been identified, such as telomere length, reactive oxygen species levels, and caveolin-1 expression status (Cho et al., 2004; Mather et al., 2011; Pandey and Rizvi, 2010).

Caveolin-1 is a membrane protein widely used to detect the aging phenotype (Cho and Park, 2005; Zhang et al., 2014). However, caveolin-1 is not only the aging marker but is important for controlling cellular signaling and homeostasis. Caveolin-1 is the main component of caveolae, which are scaffold membrane domains rich in lipid rafts that provide a platform for interactions between membrane receptors and their ligands (Capelluto, 2013). Moreover, caveolin-1 is a tumor suppressor that regulates various cellular processes, such as vesicular transport, cellular proliferation, apoptosis, and cholesterol homeostasis (Wang et al., 2013). In addition, caveolin-1 has a vital role in host defense, inflammation, and host/pathogen interactions (Lim et al., 2010; 2014; Mirza et al., 2010). However, the role of caveolin-1 in innate immune systems is not well known.

Toll like receptors (TLRs) are the best characterized among the four major classes of pattern recognition receptors that play a central role in innate immunity (Delgado and Deretic, 2009). Research on TLRs has focused on their immune function and has been extended to aging and cancer (Qian et al., 2012; Rakoff-Nahoum and Medzhitov, 2009). TLR signaling is defective in macrophages or dendritic cells during normal aging (Dunston and Griffiths, 2010; Panda et al., 2010). However, our group and Qian's group recently showed that TLR5 in peritoneal macrophages and monocytes from older donors, respectively are conserved during aging (Lim et al., 2015; Qian et al., 2012) . Importantly, our group demonstrated that caveolin-1 expression increases dramatically and that caveolin-1 directly binds TLR5 leading to a signaling cascade and the production of proinflammatory cytokines in aged macrophages (Lim et al., 2015). However, we have not yet fully documented the functions of caveolin-1 for maintaining TLR5 expression. Here, we further elucidated the relationship between TLR5 and caveolin1 at the transcriptional and translational levels using human cells and tissues from aged or caveolin-1 knockout mice. The present data suggest that caveolin-1 is a crucial regulator for maintaining and controlling TLR5 expression.

\section{MATERIALS AND METHODS}

\section{Reagents}

Monoclonal anti-caveolin-1 antibody (610406) was purchased 
from BD Transduction Laboratories (USA). Polyclonal antiTLR4 (IMG-587A) and anti-TLR5 (IMG-580) were acquired from Imgenex (USA). Anti-SAPK/JNK (\#9252) and antiphospho-SAPK/JNK (\#9251) were purchased from Cell Signaling Technology (USA). Anti- $\beta$-actin (sc-47778), anti-Erk1 (sc-94G), anti-p-Erk (sc-7383), anti-p38 $\alpha$ (sc-535-G), anti-p-p38 (sc7973), and secondary horseradish peroxidase-conjugated antigoat (sc-2020), anti-rabbit (sc-2030), and anti-mouse (sc-2302) antibodies were purchased from Santa Cruz Biotechnology (USA). Lipofectamine 2000 was obtained from Invitrogen (USA). Other biochemical reagents were purchased from Sigma Chemical (USA).

\section{Cell culture}

Human diploid fibroblasts (HDF) were ordered from the Institutional Research Committee of Seoul National University Hospital. The cells were isolated from the foreskin of a 6-year-old boy and identified by using the approval protocol of the Institutional Research Committee of Seoul National University Hospital, as described previously (Cho et al., 2003). The HDF were maintained in $100 \mathrm{~mm}$ plates in Dulbecco's modified Eagle's medium supplemented with $10 \%$ fetal bovine serum (FBS) and $1 \%$ penicillin/streptomycin. The HDF were subcultured at a ratio of 1:4. We defined non-senescent cells (NS-HDF) as those resulting from fewer than 25 population doublings and senescent cells (S-HDF) as those resulting from more than 60 population doublings. Human cervical cancer (HeLa) cells were purchased from American Type Culture Collection (ATCC CCL2, USA) and maintained like the HDF. All cell culture reagents were acquired from Gibco-BRL (USA).

\section{Animals}

Young female C57BL/6J mice (8-10 weeks old) and caveolin-1 knockout mice (8-10 weeks old) were purchased from The
Jackson Laboratory (USA). The knockout mice were inbred by homozygous mating. Aged female C57BL/6J mice (24 months old) were kindly provided by Dr. Jang (Korea Basic Science Institute, Korea). All mice were maintained on a standard diet in a specific-pathogen free facility at the Clinical Vaccine R\&D Center of Chonnam National University. All animal-related experiments were designed in accordance with the guidelines of the Institutional Animal Care and Use Committee of Chonnam National University Medical School.

\section{Lentivirus transduction}

The lentivirus was transduced as described previously (Lim et al., 2014). Briefly, caveolin-1 gene of human origin was cloned into the lentiviral transfer vector (pLentiH1.4-monomerRFP) to make a lentivirus for overexpressing caveolin- 1 protein by cotransfection of the transfer vector with a glycoprotein of the vesicular stomatitis virus (VSV-G) expression vector and a gagpol expression vector into 293T cells. The viruses were collected and transduced into HeLa and NS-HDF cells. RFP or RFP/Cav1 gene with a cytomegalovirus (CMV) promoter was integrated into the host cell genome and the protein expression was controlled by using CMV promoter. To infect the cells with lentivirus, HeLa and NS-HDF cells were seeded in 6-well plates, cultured to $60-70 \%$ confluence, and infected with $1 \mathrm{ml}$ lentivirus for $8 \mathrm{~h}$. After incubation, the cells were supplied with growth medium containing $10 \%$ FBS and harvested 48 or $72 \mathrm{~h}$ later for further assay.

RNA extraction and reverse transcription-polymerase chain reaction ( $R T-P C R)$

Total RNA was extracted from cells and tissues using Qiazol reagent (QIAGEN Sciences \#79306, Germany). cDNA was synthesized using the PrimeScript $1^{\text {st }}$ strand cDNA synthesis kit (TaKaRa Bio., Inc., \#6110A, Japan) according to the manufac-
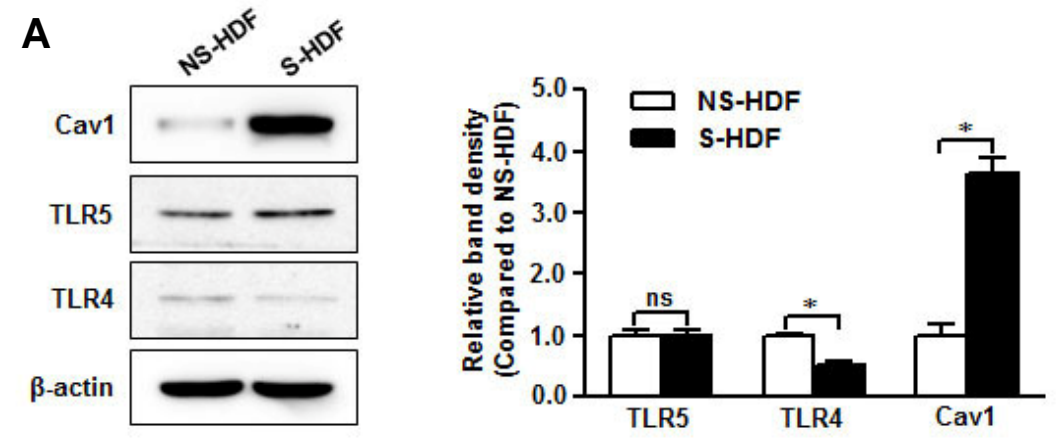

B

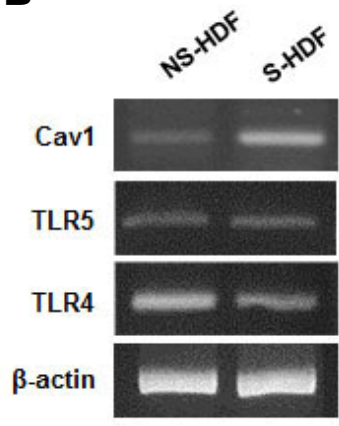

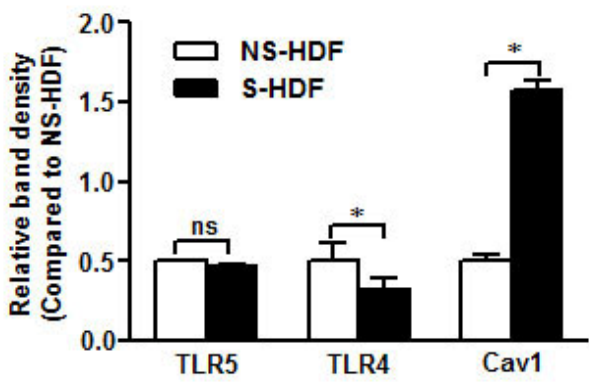

Fig. 1. TLR5 is conserved in senescent non-immune cells. RNA and protein were isolated from NS-HDF and S-HDF cells. (A) Caveolin-1, TLR4, and TLR5 protein expression was detected by Western blot with specific antibodies. (B) Caveolin-1, TLR4, and TLR5 mRNA levels were analyzed by RTPCR with specific primers. The $\beta$-actin was used as the loading control for both RT-PCR and Western blotting. The relative density of protein expression and mRNA levels were normalized to $\beta$-actin and represented by quantitative graphs. Data are presented as mean $\pm S D$ from five independent experiments; $* p<0.05$; ns, not significant. 
A
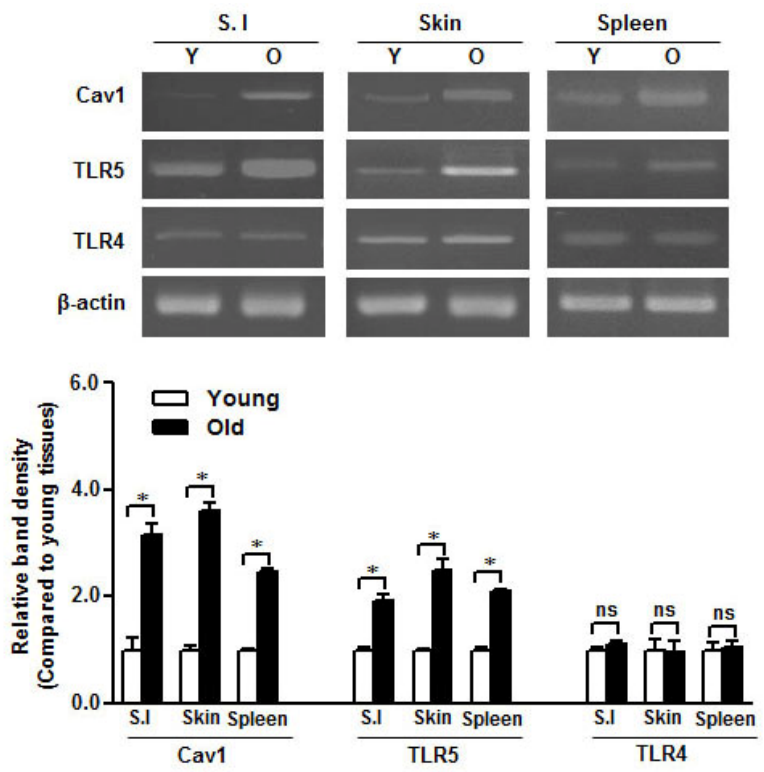

B
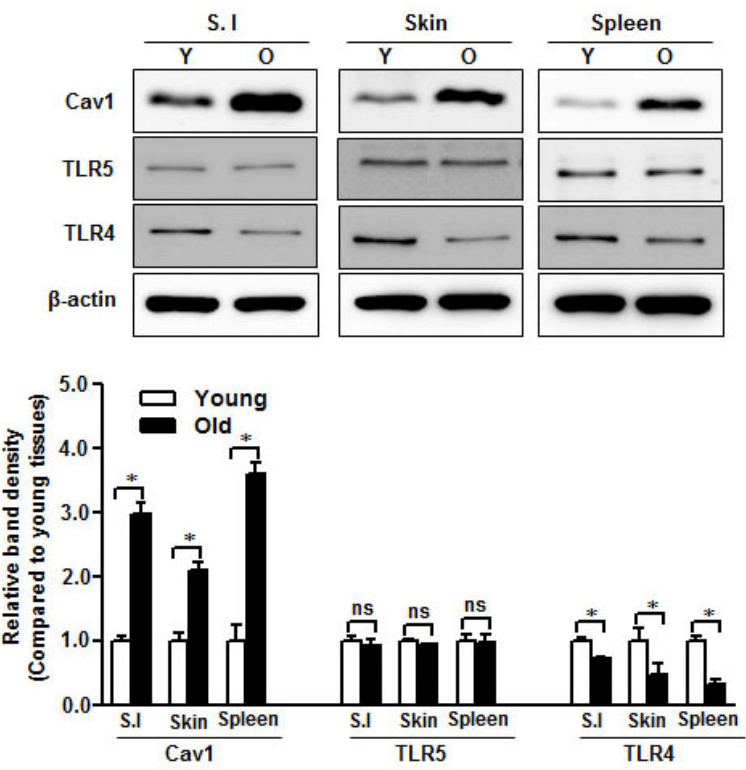

Fig. 2. TLR5 is preserved in aged mice tissues. RNA and protein were isolated from small intestine (S.I.), skin, and spleen of young (Y) and aged (O) mice. (A) Caveolin-1, TLR4, and TLR5 mRNA levels were quantified by RT-PCR with specific primers. (B) Caveolin-1, TLR4, and TLR5 protein expression was detected by Western blotting with specific antibodies. The $\beta$-actin was used as the loading control for both RTPCR and Western blotting. The relative density of protein expression and mRNA levels were normalized to $\beta$-actin and represented by quantitative graphs. Data are presented as mean \pm SD from five independent experiments ( $n=3$ mice/group); $* p<0.05$; ns, not significant.

turer instructions. The TLR4, TLR5, and caveolin-1(Cav1) primer sequences were as follows: $\beta$-actin sense TGG AAT CCT GTG GCA TCC ATG AAA C, $\beta$-actin anti-sense TAA AAC GCA GCT CAG TAA CAG TCC G; TLR4 sense AGC CTA AGC CAC CTC TCT ACC T, TLR4 anti-sense AGC CTA AGC CAC CTC TCT ACC T; Cav1 human sense GAG CTG AGC GAG AAG CAA GT, Cav1 human anti-sense TCC CTT CTG GTT CTG CAA TC; TLR5 human sense TGC CAC TGT TGA GTG CAA GTC, TLR5 human anti-sense ACC TGG AGA AGC CGA AGG TAA G; Cav1 mouse sense AGC CGC GTC TAC TCC ATC TA, Cav1 mouse anti-sense CAT CAG CAC GCA GAA AGA GA; TLR5 mouse sense GGA CAC TGA AGG ATT TGA AGA TG, TLR5 mouse anti-sense GGA CCA TCT GTA TGC TTG GAA TA. The PCR conditions were: pre-denaturation at $94^{\circ} \mathrm{C}$ for 3 min followed by 20 ( $\beta$-actin) and 25 (Cav1, TLR4, and TLR5) cycles of denaturation at $94^{\circ} \mathrm{C}$ for $30 \mathrm{~s}$, annealing at $58^{\circ} \mathrm{C}$ (Cav1), $52^{\circ} \mathrm{C}$ (TLR5) or $59^{\circ} \mathrm{C}$ (TLR4) for $30 \mathrm{~s}$, extension at $72^{\circ} \mathrm{C}$ for $30 \mathrm{~s}$, and post-extension at $72^{\circ} \mathrm{C}$ for 10 min using a TaKaRa PCR Thermal Cycler. PCR products were separated on $3 \%$ agarose gel and stained with ethidium bromide.

\section{Western blot analysis}

Western blot analysis was performed as described previously (Lim et al., 2010). Briefly, the cells and tissues were collected, and proteins were isolated by adding lysis buffer [50 mM Tris- $\mathrm{HCl}$ (pH 7.5), $150 \mathrm{mM}$ sodium chloride, $1 \mathrm{mM}$ ethylenediaminetetraacetic acid, $60 \mathrm{mM}$ octyl $\beta$-D-glucopyranoside, $1 \mathrm{mM}$ phenylmethylsulfonyl fluoride, $10 \mu \mathrm{g} / \mathrm{ml}$ aprotinin, $10 \mu \mathrm{g} / \mathrm{ml}$ leupeptin, 50 $\mathrm{mM}$ sodium fluoride, and $1 \mathrm{mM}$ sodium orthovanadate] and sonicated briefly. The proteins were subjected to sodium dodecyl sulfate polyacrylamide gel electrophoresis. The separated proteins were transferred to polyvinylidene fluoride membranes (Pall Co., USA), and the membranes were incubated with anti-Cav1, anti-TLR4, anti-TLR5, and anti- $\beta$-actin antibodies in a cold room overnight and then with peroxidase-conjugated secondary antibodies at room temperature for $1 \mathrm{~h}$. Protein expression was visualized using an enhanced chemiluminescence detection kit (Amersham ECL kit; GE Healthcare, UK).

\section{Statistical analysis}

The statistical analysis was performed using Prism 5 (GraphPad, Inc., USA). The gene and protein expression band density was quantified using ImageJ software (National Institutes of Health, USA). Differences between experimental groups were analyzed using the non-parametric Mann-Whitney test and were considered significant for $p$ values $<0.05$. Data are presented as mean \pm standard deviation (SD) of at least five independent experiments.

\section{RESULTS}

TLR5 is preserved in senescent cells and aged tissues We first tested basal caveolin-1, TLR4, and TLR5 protein expression in NS-HDF and S-HDF. As an aging maker, the caveolin-1 protein was increased significantly in S-HDF compared to that in NS-HDF, and the TLR5 protein was well expressed in both cells but expression of the TLR4 protein was diminished in S-HDF (Fig. 1A). TLR5, TLR4, and caveolin-1 mRNA levels were consistent with protein expression (Fig. 1B) and showed that cellular senescence was affected by different patterns caveolin-1 and TLR4 mRNA expression but was not influenced by TLR5 mRNA level.

To further investigate the relationship between TLR5 and caveolin-1 in aging, we collected skin, spleen, and small intestine from young and aged mice to analyze mRNA and protein expression levels in those tissues. We found that TLR5 mRNA level was increased in aged tissues, similar to caveolin-1 mRNA (Fig. 2A). The TLR5 protein was similarly expressed in 

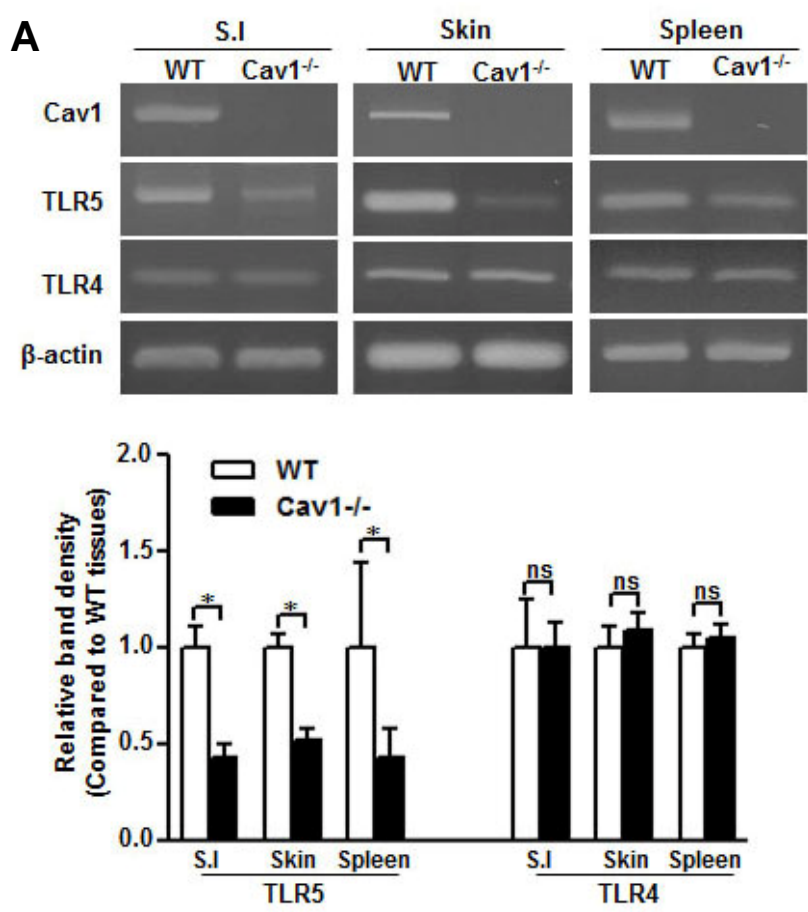
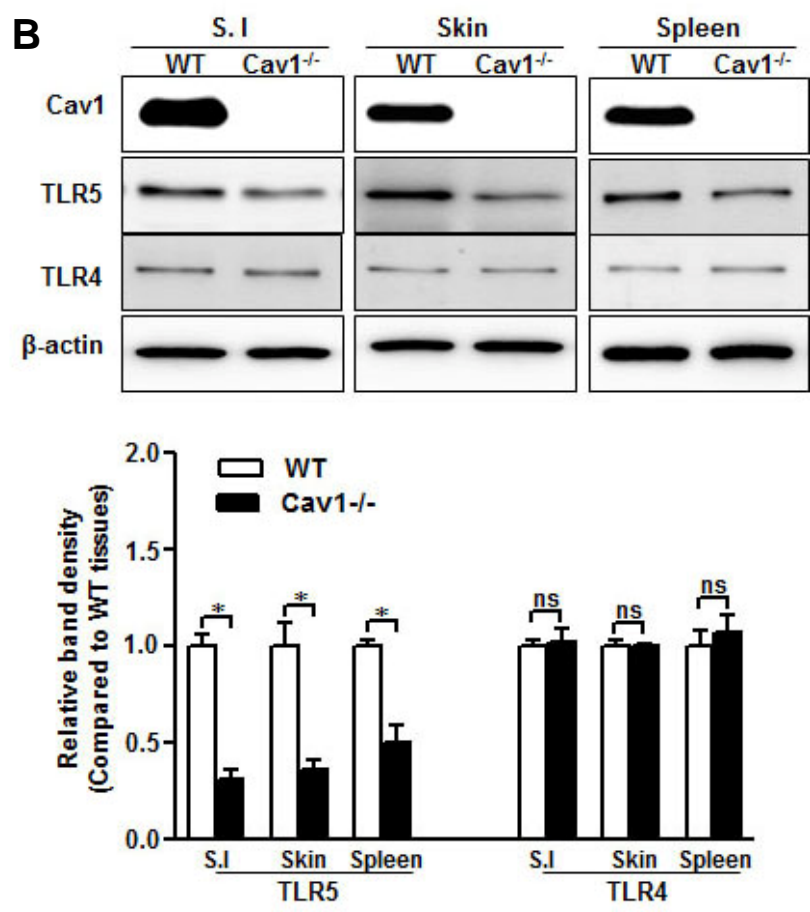

Fig. 3. TLR5 expression decreases in caveolin-1 knockout mice. RNA and protein were isolated from small intestine (S.I.), skin, and spleen of wild type (WT) and caveolin-1 knockout (Cav1 ${ }^{-1}$ ) mice. (A) Caveolin-1, TLR4, and TLR5 mRNA levels were analyzed by RT-PCR with specific primers. (B) Caveolin-1, TLR4, and TLR5 protein expression was detected by Western blotting with specific antibodies. The $\beta$-actin was used as the loading control for both RT-PCR and Western blotting. Relative density of protein expression and mRNA levels were normalized to $\beta$ actin and represented by quantitative graphs. Data are presented as mean \pm SD from five independent experiments $(\mathrm{n}=3$ mice/group); $* p<$ 0.05 ; ns, not significant.

both young and aged tissues, which differed from the caveolin1 expression pattern in those tissues (Fig. 2B). TLR4 mRNA level was not affected by aging (Fig. 2A) but TLR4 protein expression was decreased in aged tissues (Fig. 2B).

Taken together, TLR5 was preserved in senescent cells and aged tissues with abundant caveolin-1 expression.

\section{TLR5 expression is affected by caveolin-1 status}

To clarify the role of caveolin-1 in the preservation of TLR5, we examined TLR5 expression in several tissues from caveolin-1 knockout $\left(\mathrm{Cav1}^{-{ }^{--}}\right)$mice. Interestingly, TLR5 mRNA was decreased significantly in tissues from Cav $1^{-1-}$ mice compared with those from normal mice, whereas TLR4 mRNA level was not affected by the caveolin-1 genetic deletion (Fig. 3A). Moreover, mRNA level was correlated with protein expression, demonstrating that TLR5 but not TLR4 was decreased in Cav1 ${ }^{--}$tissues (Fig. 3B). This result indicates that caveolin-1 may regulate TLR5 through both transcriptional and translational processes.

\section{Caveolin-1 may be a crucial regulator of TLR5 expression} To elucidate the function of caveolin-1 in the regulation of TLR5, caveolin-1 was overexpressed in NS-HDF and HeLa cells with low level of caveolin-1. Caveolin-1 over-expression increased TLR5 mRNA level in both cell types but not that of TLR4 (Fig. 4A). TLR5 protein expression was increased significantly in caveolin-1 overexpressed cells than that in control cells, while the TLR4 protein was not influenced by caveolin-1 overexpression (Fig. 4B). These results indicate that both TLR5 mRNA and protein were affected by exogenous caveolin-1 expression and that caveolin-1 may be a novel regulator of TLR5 to maintain the receptor during aging.

\section{Molecular mechanism of caveolin-1-dependent TLR5 expression}

Previously, we demonstrated that caveolin-1 directly interacts with TLR5 and mediates the signaling cascade via flagellin stimulation (Lim et al., 2015). To understand the function of caveolin-1 in the conservation of TLR5, we determined whether caveolin-1 expression affected TLR5 stability. We blocked new protein synthesis in HeLa cells and NS-HDF with cycloheximide to determine a $24 \mathrm{~h}$ half-life for the TLR5 protein in both cell types (Figs. 5A and 5B). However, we did not observe significant degradation of TLR5 in caveolin-1 overexpressed HeLa (Fig. 5A) or caveolin-1 overexpressed NS-HDF (Fig. 5B) after exposure to cyclohexamide for up to $36 \mathrm{~h}$. Thus, these data suggest that caveolin-1 functioned as a scaffold molecule to prolong the half-life of the TLR5 protein. Recently it has been reported that expression of TLRs is regulated by the mitogenactivated protein kinase (MAPK) signaling cascade. We also found that overexpression of caveolin-1 in HeLa cells induced phosphorylation of extracellular signal-regulated kinase ( $p$-Erk) and stress-activated kinases c-Jun N-terminal kinase ( $p$ SAPK/JNK); however, it did not influence phosphorylation of p38 kinase (p-p38) (Fig. 5C). Hence, the increment of caveolin1-activated MAPK signaling cascades causes phosphorylation of downstream molecules Erk and SAPK/JNK, leading to an increase in TLR5 mRNA expression. 
$\boldsymbol{A}$
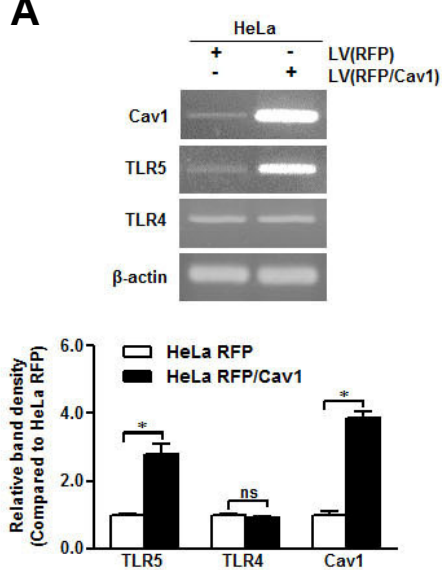

$\boldsymbol{B}$
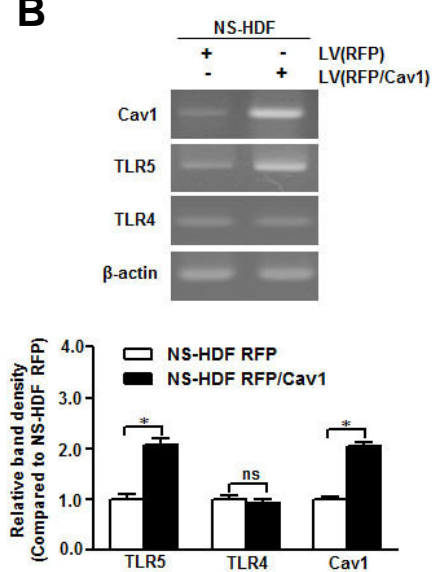

C
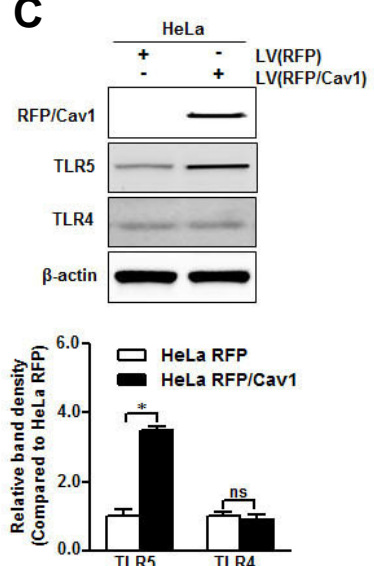
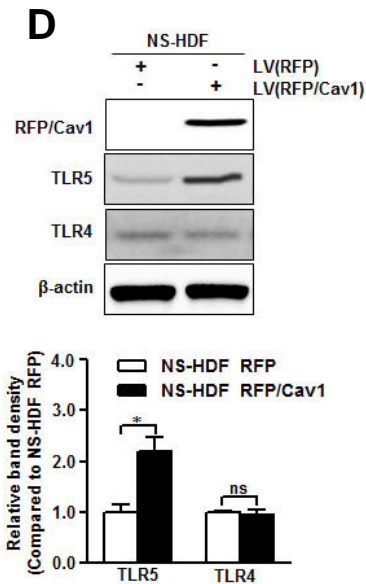

Fig. 4. Caveolin-1 may be a crucial TLR5 mediator. Caveolin-1 was overexpressed in HeLa and NS-HDF cells using a lentivirus (LV) carrying RFP-caveolin-1 (RFP/Cav1). (A, B) Caveolin-1, TLR4, and TLR5 mRNA levels in caveolin-1 over-expressed HeLa and NS-HDF were analyzed by RT-PCR with specific primers, respectively. (C, D) Caveolin-1, TLR4, and TLR5 protein expression in caveolin-1 overexpressed HeLa and NS-HDF was detected by Western blotting with specific antibodies. The $\beta$-actin was used as the loading control for both RT-PCR and Western blotting. The relative density of protein expression and mRNA levels were normalized to $\beta$-actin and represented by quantitative graphs. Data are presented as mean \pm SD from five independent experiments; $* p<0.05$; ns, not significant.

$\boldsymbol{A}$

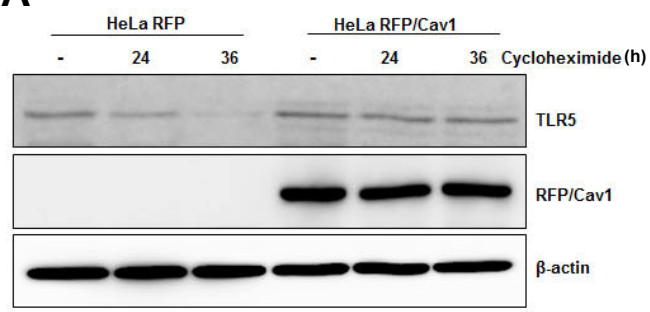

B

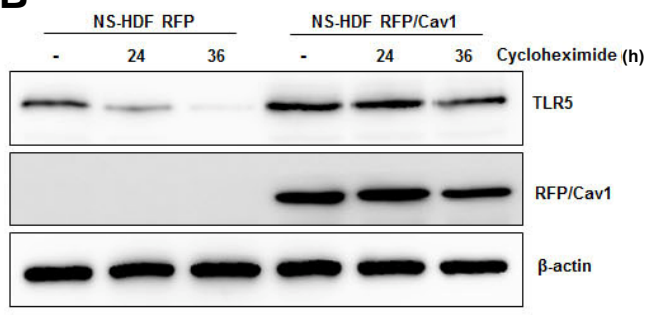

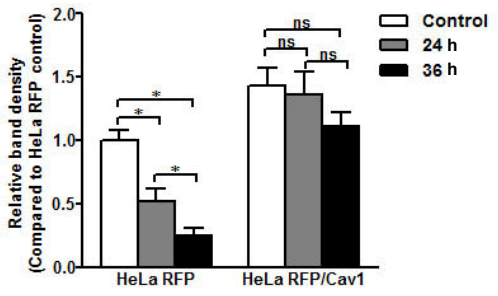

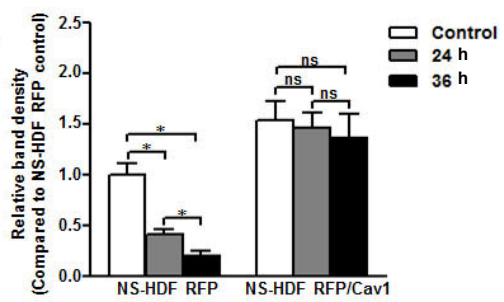

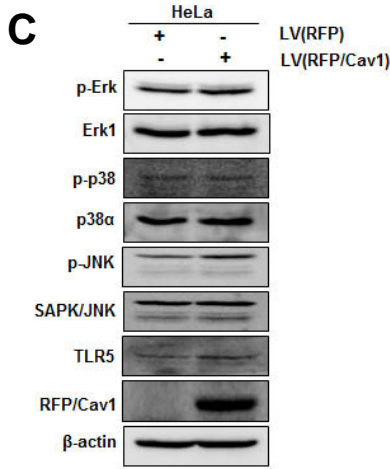

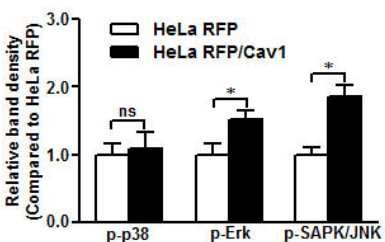

Fig. 5. Molecular mechanism of caveolin-1-dependent TLR5 expression. Caveolin-1 was overexpressed in HeLa and NS-HDF cells using a lentivirus (LV) carrying RFP-caveolin-1 (RFP/Cav1). (A, B) The protein half-life of TLR5 in caveolin-1 overexpressing HeLa and NS-HDF after treatment with $100 \mu \mathrm{g} / \mathrm{ml}$ cycloheximide for the indicated time period was detected by Western blotting with anti-TLR5 specific antibody. (C) The proteins associated with the MAPK signaling pathway (Erk1, p-Erk, p38 $\alpha$, p-p38, SAPK/JNK, p-SAPK/JNK) were detected in caveolin-1 over-expressing HeLa cells by Western blotting using specific antibodies. The $\beta$-actin was used as the loading control. The relative density of protein expression was normalized to $\beta$-actin and represented by quantitative graphs. Data are presented as mean \pm SD from five independent experiments; $* p<0.05$; ns, not significant.

\section{DISCUSSION}

We have previously observed that TLR5 expression in peritoneal macrophages is conserved during aging due to direct interactions with caveolin-1 (Lim et al., 2015). This interesting result encouraged us to determine the relationship between caveolin1 and TLR5 in non-immune senescent cells that express abundant caveolin-1. Using S-HDF as the senescent cell model, we found that both TLR5 mRNA and protein were conserved in those cells as observed in older monocytes and aged macrophages (Lim et al., 2015; Qian et al., 2012), whereas TLR4 and other TLRs, mRNA and proteins were decreased dramatically.

The preservation of TLR5 in aging was further assessed by analyzing TLR5 expression in several aged tissues, including skin, spleen, and small intestine. We found that TLR5 mRNA level was enhanced in aged tissues but TLR5 protein expres- 
sion in those tissues was comparable to that in young tissues, suggesting that the TLR5 protein was conserved during aging; however, the elevated TLR5 mRNA level in aged tissues differed from the cell-based model. Cells are affected by constant paracrine and exocrine signaling required for tissue differentiation and maintenance (Weinberg, 2013), so mRNA and protein expressions in tissues may be regulate differently than that in in vitro cell culture. Thus, the difference in the TLR5 mRNA expression pattern in a cell or tissue model may be not only due to a difference in transcriptional regulation or post-transcriptional processing but also to the export rate of mature mRNA to the cytoplasm or stabilization of the mRNA itself. Although TLRs have widely investigated in various cell types and diseased tissues (Applequist et al., 2002; Betanzos-Cabrera et al., 2012; So and Ouchi, 2010), the TLRs expression in aged tissues is largely unknown. Only one group has reported that TLR1, TLR2, TLR4, TLR5, and TLR7 mRNA is upregulated in the aged mice brain (Letiembre et al., 2007). However, we did not observe any change in TLR4 mRNA level in aged tissues in our model but TLR5 mRNA expression was enhanced in those tissues, as reported in the aged brain (Letiembre et al., 2007). Thus, aging affected TLR5 transcription but not translation in aged tissues.

We have also suggested that the expression and activation of TLR5/MyD88 signaling in macrophages is sensitively upregulated by caveolin-1 through direct interactions but we have not investigated the TLR5 expression pattern in Cav1 $1 /$ tissues (Lim et al., 2015). In this study, several tissues from Cav1\% mice were used to determine TLR5 mRNA and protein expression. Both TLR5 mRNA and protein expression decreased in Cav1 $\%$ tissues, whereas TLR4 mRNA and protein were expressed, regardless of caveolin-1 status. Although TLR4 directly interacts with caveolin-1 and TLR4 signaling is impaired in Cav1 $1 \%$ mice through endothelial nitric oxide synthase, no evidence exists about TLR4 mRNA and protein levels in mice (Jiao et al., 2013; Mirza et al., 2010; Wang et al., 2009). We observed that both TLR4 mRNA and protein expression was not affected by genetically deleting caveolin- 1 . Therefore, we propose that the TLR4/caveolin-1 and TLR5/caveolin-1 may be regulated by different mechanisms.

The function of caveolin- 1 in the regulation of TLR5/caveolin1 was further examined in caveolin-1 over-expressing cells. Caveolin-1 expression in cancer (HeLa) and normal (NS-HDF) cells dramatically enhanced TLR5 expression at both mRNA and protein levels but no change in TLR4 occurred. Hence, caveolin- 1 not only controlled TLR5 expression at the mRNA level but also at the protein level. It has been reported that components of the MAPK signaling pathway regulate TLR mRNA (Peroval et al., 2013); hence, we checked the phosphorylation state of MAPK-related proteins in caveolin-1 overexpressing HeLa cells. We confirmed that caveolin-1 enhanced phosphorylation of Erk and SAPK/JNK proteins in the MAPK cascade. Hence, the transcriptional regulation of TLR5 might be regulated by caveolin-1 through the MAPK pathway. To elucidate the meaning of the direct interaction between caveolin-1 and TLR5, we examined whether TLR5 protein half-life was affected by caveolin- 1 status. As a result, half-life of the TLR5 protein in HeLa cells and NS-HDF was about $24 \mathrm{~h}$, which is similar to the half-life of the TLR4 protein (O'Hara et al., 2009) and the protein decreased dramatically after $36 \mathrm{~h}$. However TLR5 was not degraded significantly at $36 \mathrm{~h}$ in caveolin-1 overexpressed cells. Thus, caveolin- 1 not only enhanced TLR5 expression but also prolonged TLR5 stability. The result indicates that caveolin-1 may serve as a scaffold protein to stabilize TLR5. Taken together, our results suggest a novel function for caveolin-1 in maintaining and regulating TLR5 expression.

\section{ACKNOWLEDGMENTS}

This project was funded by the Basic Science Research Program through the National Research Foundation of Korea (NRF), which is funded by the Ministry of Education, Science and Technology (2010-0011532) and by Leading Foreign Research Institute Recruitment Program through the National Research Foundation of Korea (NRF) funded by the Ministry of Education, Science and Technology (MEST) (2011-0030034). This study was financially supported by Chonnam National University (2014-2220).

\section{REFERENCES}

Applequist, S.E., Wallin, R.P.A., and Ljunggren, H.G. (2002) Variable expression of Toll-like receptor in murine innate and adaptive immune cell lines. Int. Immunol. 14, 1065-1074.

Betanzos-Cabrera, G., Estrada-Luna, D., Belefant-Miller, H., and Cancino-Díaz, J.C. (2012). Mice fed with a high fat diet show a decrease in the expression of toll like receptor (TLR)2 and TLR6 mRNAs in adipose and hepatic tissues. Nutr. Hosp. 27, 11961203.

Campisi, J. (2013). Aging, cellular senescence, and cancer. Annu. Rev. Physiol. 75, 685-705.

Campisi, J., Andersen, J., Kapahi, P., and Melov, S. (2011). Cellular senescence: a link between cancer and age-related degenerative disease? Semin. Cancer Biol. 21, 354-359.

Capelluto, D.G.S. (2013). Lipid-mediated Protein Signaling (Springer).

Cho, K.A., and Park, S.C. (2005). Caveolin-1 as a prime modulator of aging: a new modality for phenotypic restoration? Mech. Ageing Dev. 126, 105-110.

Cho, K.A., Ryu, S.J., Park, J.S., Jang, I.S., Ahn, J.S., Kim, K.T., and Park, S.C. (2003). Senescent phenotype can be reversed by reduction of caveolin status. J. Biol. Chem. 278, 27789-27795.

Cho, K.A., Ryu, S.J., Oh, Y.S., Park, J.H., Lee, J.W., Kim, H.-P., Kim, K.T., Jang, I.S., and Park, S.C. (2004). Morphological adjustment of senescent cells by modulating caveolin-1 status. J. Biol. Chem 279, 42270-42278.

Delgado, M.A., and Deretic, V. (2009). Toll-like receptors in control of immunological autophagy. Cell Death Differ. 16, 976-983.

Dunston, C.R., and Griffiths, H.R. (2010). The effect of ageing on macrophage Toll-like receptor-mediated responses in the fight against pathogens. Clin. Exp. Immunol. 161, 407-416.

Jiao, H., Zhang, Y., Yan, Z., Wang, Z.-G., Liu, G., Minshall, R.D., Malik, A.B., and Hu, G. (2013). Caveolin-1 Tyr14 phosphorylation induces interaction with TLR4 in endothelial cells and mediates MyD88-dependent signaling and sepsis-induced lung inflammation. J. Immunol. 191, 6191-6199.

Letiembre, M., Hao, W., Liu, Y., Walter, S., Mihaljevic, I., Rivest, S., Hartmann, T., and Fassbender, K. (2007). Innate immune receptor expression in normal brain aging. Neuroscience 146, 248-254.

Lim, J.S., Choy, H.E., Park, S.C., Han, J.M., Jang, I.S., and Cho, K.A. (2010). Caveolae-mediated entry of Salmonella typhimurium into senescent nonphagocytotic host cells. Aging Cell 9, 243-251.

Lim, J.S., Shin, M., Kim, H.J., Kim, K.S., Choy, H.E., and Cho, K.A. (2014). Caveolin-1 mediates Salmonella invasion via the regulation of SopE-dependent Rac1 activation and actin reorganization. J. Infect Dis. 210, 793-802.

Lim, J.S., Nguyen, K.C.T., Nguyen, C.T., Jang, I.-S., Han, J.M., Fabian, C., Lee, S.E., Rhee, J.H., and Cho, K.A. (2015). Flagellin-dependent TLR5/caveolin-1 as a promising immune activator in immunosenescence. Aging Cell 14, 907-915.

Mather, K.A., Jorm, A.F., Parslow, R.A., and Christensen, H. (2011). Is telomere length a biomarker of aging? A Review. J. Gerontol. A Biol. Sci. Med. Sci. 66A, 202-213.

Menni, C., Kastenmüller, G., Petersen, A.K., Bell, J.T., Psatha, M., Tsai, P.-C., Gieger, C., Schulz, H., Erte, I., John, S., et al. (2013). Metabolomic markers reveal novel pathways of ageing and early development in human populations. Int. J. Epidemiol. 42, 11111119.

Mirza, M.K., Yuan, J., Gao, X.-P., Garrean, S., Brovkovych, V., Malik 
A.B., Tiruppathi, C., and Zhao, Y.-Y. (2010). Caveolin-1 deficiency dampens toll-like receptor 4 signaling through eNOS activation. Am. J. Pathol. 176, 2344-2351.

O'Hara, S.P., Small, A.J., Gajdos, G.B., Badley, A.D., Chen, X.M., and LaRusso, N.F. (2009). HIV-1 Tat protein suppresses cholangiocyte toll-like receptor 4 expression and defense against Cryptosporidium parvum. J. Infect Dis. 199, 1195-1204.

Panda, A., Qian, F., Mohanty, S., van Duin, D., Newman, F.K., Zhang, L., Chen, S., Towle, V., Belshe, R.B., Fikrig, E., et al. (2010). Age-associated decrease in TLR function in primary human dendritic cells predicts influenza vaccine response. J. Immunol. 184, 2518-2527.

Pandey, K.B., and Rizvi, S.I. (2010). Markers of oxidative stress in erythrocytes and plasma during aging in humans. Oxid. Med. Cell Longev. 3, 2-12.

Peroval, M.Y., Boyd, A.C., Young, J.R., and Smith, A.L. (2013). A critical role for MAPK signalling pathways in the transcriptional regulation of toll like receptors. PLoS One 8, e51243.

Qian, F., Wang, X., Zhang, L., Chen, S., Piecychna, M., Allore, H., Bockenstedt, L., Malawista, S., Bucala, R., Shaw, A.C., et al. (2012). Age-associated elevation in TLR5 leads to increased inflammatory responses in the elderly. Aging Cell 11, 104-110.

Rakoff-Nahoum, S., and Medzhitov, R. (2009). Toll-like receptors and cancer. Nat. Rev. Cancer 9, 57-63.

So, E.Y., and Ouchi, T. (2010). The application of Toll like receptors for cancer therapy. Int. J. Biol. Sci. 6, 675-681.

Wang, X.M., Kim, H.P., Nakahira, K., Ryter, S.W., and Choi, A.M.K. (2009). The heme oxygenase-1/carbon monoxide pathway suppresses TLR4 signaling by regulating the interaction of TLR4 with caveolin-1. J. Immunol. 182, 3809-3818.

Wang, X.X., Wu, Z., Huang, H.F., Han, C., Zou, W., and Liu, J. (2013). Caveolin-1, through its ability to negatively regulate TLR4 is a crucial determinant of MAPK activation in LPS-challenged mammary epithelial cells. Asian Pac. J. Cancer Prev. 14, 22952299.

Weinberg, R. (2013). The Biology of Cancer (Taylor \& Francis Group).

Zhang, L., Hu, X.Z., Li, X., Li, H., Smerin, S., Russell, D., and Ursano, R.J. (2014). Telomere length - a cellular aging marker for depression and post-traumatic stress disorder. Med. Hypotheses 83, 182-185. 